\title{
RHF AND DFT STUDY OF THE OPTIMIZED MOLECULAR STRUCTURE AND ATOMIC CHARGE DISTRIBUTION OF PENTACENE
}

\author{
*Galadanci, G. S.M., Chifu, E. Ndikilar, Safana A.A. and Sabiu, S. A \\ Physics Department, Federal University Dutse, P.M.B 7156, Dutse, Jigawa State Nigeria \\ *Email: gsmgalad@gmail.com Tel: +2348037260380
}

\begin{abstract}
Restricted HartreeFock (RHF) and Density Functional Theory (DFT) studies were carried out on the organic semi conductor material Pentacene. 6-31G and 6-31G* basis sets were used to optimize the molecule and compute the charge distribution at both levels of theory. The results show that the Carbon-Hydrogen bonds in the molecule are the shortest and strongest with average bond length of 1 $A$. The $C$ - $C$ bonds were the longest and weakest. On the average the bond angles were all above 90 degrees, giving the molecule a fairly symmetric structure. The bulk positive charges in Pentacene were predicted to reside mostly in the Hydrogen atoms and the Carbon atoms were averagely electronegative in this molecule.
\end{abstract}

\section{INTRODUCTION}

Organic field-effect transistors (FETs) have attracted considerable attention due to their potential in organic thin film transistors (OTFTs) with large-area of application that are mechanically flexible, lightweight (as switching devices for logic gates and memory arrays), organic light emitting diodes (OLEDs), solar cells and low-cost devices (Kitamura,Arakawa, 2008; Hwan et al.2004; Byoungnan,2008). Organic semiconductors are materials with semiconducting properties.These include Polycyclic aromatic hydrocarbons such as anthracene, rubrene and pentacene. Among many organic semiconductors, pentacene has been reported to be the most promising applicant, because of its high hole mobility up to $5.5 \mathrm{~cm}^{2} / \mathrm{Vs}$ that exceed amorphous Silicon (Hwan et al, 2004). Pentacene is a linear acene consisting of five benzene rings with molecular formula $\mathrm{C}_{22} \mathrm{H}_{14}$, molar mass $278.36 \mathrm{~g} / \mathrm{mol}$,density $1.3 \mathrm{~g} / \mathrm{cm}^{3}$ and melting point of > $300^{\circ} \mathrm{C}$; sublimes at $372^{\circ} \mathrm{C}$ which behaves as a p-type semiconductor. These kind of materials are important in electrical applications as their band gap is controlled by selecting the number of aromatic rings that is the more rings, the smaller the band gap. Crystallized pentacene has triclinic lattice structure comprising of. Moreover this compound is purple powder which generate exciton upon absorption of Ultraviolet(UV) or visible light that makes it very sensitive to oxidation, this is the reason why the compound degrades upon exposure to air or light (Dan, 2005). With these remarkable properties Pentacene and other derivatives of benzene attract both experimental and theoretical research.

There has been a report on organic $p-i-n$ homojunctions composed of differently doped vacuum-deposited pentacene layers. Where remarkably high built-in voltage of $1.65 \mathrm{~V}$ was observed; moreover an analysis of the current-voltage characteristics under dark and illuminated conditions reveals that the opencircuit voltage is directly related to the built-in voltage whereby the recombination process is influenced by the distinct charge transport properties of electrons and holes in the pentacene film (Harada, 2008). Furthermore, investigation have shown that doping pentacene films with iodine and alkaline metals help in achieving maximum conductivity of $150 \mathrm{~S} \mathrm{~cm}^{-1}$ for highly ordered films heavily doped with iodine, and 2.8 $\mathrm{S} \mathrm{cm}^{-1}$ for a rubidium-doped film, which turned the film into an n-type Semiconductor(Dan, 2005). Other experimental research include Channel Formation in Single-Mono layer Pentacene Thin Film Transistors, Orientation of Pentacene molecules on $\mathrm{SiO}_{2}$ by Byoungnam Park(Byoungnan,2008); Modelling the UV/visible spectrum of etrakis (phenylethynyl) benzene by Denis Jacquemin(Jaquemin et al, 2008] Molecular structure, vibrational, UV and NBO analysis of 4-chloro7-nitrobenzofurazan by DFT calculations by M. Kurta (Kurta et al;']aquemin et al; 2005).

Nowadays, the molecular modelization techniques offer a competitive alternative for the interpretation of experimental data arising from industrial interest and applications (Jaquemin et al, 2005).In this article, the Gaussian software is used to compute the optimum electronic structure ofpentaceneand its atomic charge distribution(James, Jan et al, 1991).

\section{COMPUTATIONAL METHOD}

The molecular structure of pentacene is modeled with the Gaussian03 package. Geometric optimization of the structure is performed at Restricted HartreeFock (RHF) and Density Functional Theory (DFT) levels of theory using the basis sets6-31G and 6-31G*.Geometry optimizations usually attempt to locate minima on the potential energy surface, thereby predicting equilibrium structures of molecular systems. At the minima, the first derivative of the energy (gradient) is zero. Since the gradient is the negative of the forces, the forces are also zero at such a point (stationary point). In Gaussian, a geometry optimization begins at the molecular structure specified at the input (in this case from GaussView) and steps along the potential energy surface. 
It computes the energy and gradient at that point, and determines which direction to make the next step. The gradient indicates the direction along the surface in which the energy decreases most rapidly from the current point as well as the steepness of that slope. The optimized parameters are the bond lengths (in Angstrom), the bond angles and the dihedral angles (in degrees) for the optimized molecular structure. Atoms in the molecule are numbered according to their order in the molecule specification section of the input. The structures are refined further using Density Functional Theory which is a cost effective method for inclusion of electron correlations with the three-parameter density functional generally known as Becke3LYP (B3LYP).

\section{RESULTS AND DISCUSSION}

\section{Optimized Bond lengths}

The optimized structure of pentacene is shown in Fig. 1 and the optimized bond lengths at different levels of theory are shown in Table 1.In molecular geometry, the bond length (or bond distance) is the average distance between nuclei of two bonded atoms in a molecule. Bond length is inversely related to bond strength and bond dissociation energy. The predicted shortest bond lengths are all in the vicinity of $1 \AA$ and these are the C$H$ bonds: $R(2,17), R(7,16), R(8,19), R(13,20), R(14,21)$, $R(15,22) ， R(23,25), \quad R(26,29) ， R(27,31) ， R(30,34)$ ， $R(32,35)$ and $R(33,36)$. Thus, since these $\mathrm{C}-\mathrm{H}$ bonds are shortest it implies they are the strongest bonds in pentacene molecule that will require much energy to be broken. On the other hand, the longest and weakest bonds with bond lengths close to $1.5 \AA$ are the following C-C bonds: $R(1,6), R(3,4), R(9,10), R(9,15), R(10,11)$, $R(13,14), R(24,27), R(24,28), R(28,32)$ and $R(30,33)$. These are all bonds within the fused benzene rings and hence it can be predicted that due to the free electron movements within the rings, the bonds are relatively weaker compared to the $\mathrm{C}-\mathrm{H}$ bonds. On the average, the $6-31 G^{*}$ basis set results are relatively higher compared to the 6-31G basis set results at both levels of theory. Interestingly, the RHF and DFT results for 631G* are the same indicating that electron correlation has very little effect on the bond lengths using this basis set; however, for 6-31G basis set, electron correlation has an effect of expanding the bond and thus the DFT results are slightly higher than those at RHF level of theory.

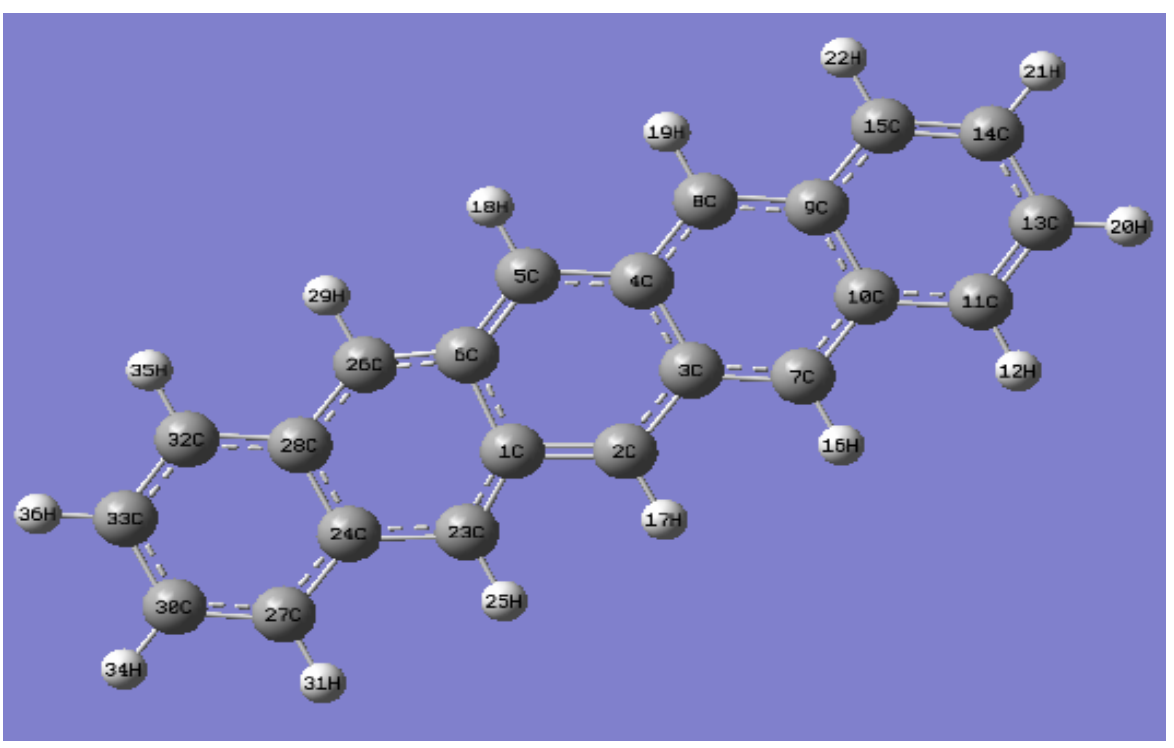

Fig.1: Optimized Structure of Pentacene 
Table 1: Optimized bond lengths $(\AA)$ of pentacene molecule

\begin{tabular}{|c|c|c|c|c|}
\hline $\begin{array}{l}\text { Geometrical } \\
\text { parameter } \\
\text { Bond lenth }(\bar{A})\end{array}$ & $\begin{array}{l}\text { RHF/6-31G } \\
\text { Gas }\end{array}$ & $\begin{array}{l}\text { RHF/6-31G* } \\
\text { Gas }\end{array}$ & $\begin{array}{l}\text { B3LYP/6-31G } \\
\text { Gas }\end{array}$ & $\begin{array}{l}\text { B3LYP/6-31G* } \\
\text { Gas }\end{array}$ \\
\hline$R(1,2)$ & 1.3930 & 1.4131 & 1.4056 & 1.4131 \\
\hline$R(1,6)$ & 1.4371 & 1.5057 & 1.4605 & 1.5057 \\
\hline $\mathrm{R}(1,23)$ & 1.4180 & 1.3733 & 1.4174 & 1.3733 \\
\hline$R(2,3)$ & 1.3929 & 1.4328 & 1.4053 & 1.4328 \\
\hline $\mathrm{R}(2,17)$ & 1.0745 & 1.1005 & 1.0870 & 1.1005 \\
\hline$R(3,4)$ & 1.4371 & 1.4288 & 1.4606 & 1.4288 \\
\hline$R(3,7)$ & 1.4181 & 1.3991 & 1.4176 & 1.3991 \\
\hline$R(4,5)$ & 1.3929 & 1.4328 & 1.4053 & 1.4328 \\
\hline$R(4,8)$ & 1.4181 & 1.3991 & 1.4176 & 1.3991 \\
\hline$R(5,6)$ & 1.3930 & 1.3646 & 1.4056 & 1.3646 \\
\hline$R(5,18)$ & 1.0745 & 1.1005 & 1.0870 & 1.1005 \\
\hline$R(6,26)$ & 1.4180 & 1.3733 & 1.4174 & 1.3733 \\
\hline $\mathrm{R}(7,10)$ & 1.3693 & 1.3991 & 1.3926 & 1.3991 \\
\hline$R(7,16)$ & 1.0746 & 1.1010 & 1.0871 & 1.1010 \\
\hline$R(8,9)$ & 1.3693 & 1.3991 & 1.3925 & 1.3991 \\
\hline $\mathrm{R}(8,19)$ & 1.0746 & 1.1010 & 1.0871 & 1.1010 \\
\hline $\mathrm{R}(9,10)$ & 1.4464 & 1.4287 & 1.4587 & 1.4287 \\
\hline $\mathrm{R}(9,15)$ & 1.4459 & 1.4328 & 1.4385 & 1.4328 \\
\hline $\mathrm{R}(10,11)$ & 1.4459 & 1.4328 & 1.4385 & 1.4328 \\
\hline $\mathrm{R}(11,12)$ & 1.0738 & 1.1005 & 1.0864 & 1.1005 \\
\hline $\mathrm{R}(11,13)$ & 1.3452 & 1.3646 & 1.3698 & 1.3646 \\
\hline$R(13,14)$ & 1.4421 & 1.4258 & 1.4353 & 1.4258 \\
\hline$R(13,20)$ & 1.0729 & 1.1000 & 1.0853 & 1.1000 \\
\hline$R(14,15)$ & 1.3452 & 1.3647 & 1.3698 & 1.3647 \\
\hline$R(14,21)$ & 1.0729 & 1.1000 & 1.0853 & 1.1000 \\
\hline$R(15,22)$ & 1.0738 & 1.1005 & 1.0864 & 1.1005 \\
\hline$R(23,24)$ & 1.3694 & 1.4726 & 1.3928 & 1.4726 \\
\hline$R(23,25)$ & 1.0746 & 1.1003 & 1.0871 & 1.1003 \\
\hline$R(24,27)$ & 1.4458 & 1.4220 & 1.4384 & 1.4220 \\
\hline$R(24,28)$ & 1.4463 & 1.4189 & 1.4586 & 1.4189 \\
\hline$R(26,28)$ & 1.3694 & 1.4221 & 1.3928 & 1.4221 \\
\hline$R(26,29)$ & 1.0746 & 1.1003 & 1.0871 & 1.1003 \\
\hline $\mathrm{R}(27,30)$ & 1.3453 & 1.3728 & 1.3699 & 1.3728 \\
\hline$R(27,31)$ & 1.0738 & 1.1003 & 1.0864 & 1.1003 \\
\hline $\mathrm{R}(28,32)$ & 1.4458 & 1.4217 & 1.4384 & 1.4217 \\
\hline$R(30,33)$ & 1.4421 & 1.4160 & 1.4351 & 1.4160 \\
\hline$R(30,34)$ & 1.0729 & 1.1000 & 1.0853 & 1.1000 \\
\hline $\mathrm{R}(32,33)$ & 1.3453 & 1.3733 & 1.3699 & 1.3733 \\
\hline$R(32,35)$ & 1.0738 & 1.1002 & 1.0864 & 1.1002 \\
\hline$R(33,36)$ & 1.0729 & 1.1000 & 1.0853 & 1.1000 \\
\hline
\end{tabular}

\section{Optimized Bond Angles}

The average angle between the orbital's of the central atom containing the bonding electron pairs in the molecular structure is known as the bond angle between the atoms. The bond angle gives an idea about the distribution of orbital's about the central atom in a molecule. Table 2 shows the optimized bond angles of pentacene at RHF and DFT levels of theory using 6$31 \mathrm{G}$ and $6-31 \mathrm{G}^{*}$ basis sets. 
Bajopas Volume 8 Number 2 December, 2015

Table 2: Optimized bond angles $\left({ }^{\circ}\right)$ of pentacene molecule

Geometrical parameter RHF/6-31G Gas RHF/6-31G* Gas

B3LYP/6-31G Gas B3LYP/6-31G* Gas

\begin{tabular}{|c|c|c|c|c|}
\hline$A(2,1,6)$ & 119.1271 & 112.1259 & 118.9965 & 112.1259 \\
\hline$A(2,1,23)$ & 122.2267 & 114.5151 & 122.2946 & 114.5151 \\
\hline$A(6,1,23)$ & 118.6462 & 130.1766 & 118.7089 & 130.1766 \\
\hline$A(1,2,3)$ & 121.7445 & 125.3768 & 122.0032 & 125.3768 \\
\hline$A(1,2,17)$ & 119.1260 & 116.5158 & 118.9912 & 116.5158 \\
\hline$A(3,2,17)$ & 119.1296 & 118.0813 & 119.0057 & 118.0813 \\
\hline$A(2,3,4)$ & 119.1285 & 118.7901 & 119.0004 & 118.7901 \\
\hline$A(2,3,7)$ & 122.2270 & 121.7006 & 122.2963 & 121.7006 \\
\hline$A(4,3,7)$ & 118.6445 & 119.5092 & 118.7032 & 119.5092 \\
\hline$A(3,4,5)$ & 119.1284 & 118.7868 & 119.0013 & 118.7868 \\
\hline$A(3,4,8)$ & 118.6445 & 119.5145 & 118.7022 & 119.5145 \\
\hline$A(5,4,8)$ & 122.2270 & 121.6986 & 122.2966 & 121.6986 \\
\hline$A(4,5,6)$ & 121.7445 & 120.7650 & 122.0034 & 120.7650 \\
\hline$A(4,5,18)$ & 119.1295 & 118.0759 & 119.0099 & 118.0759 \\
\hline$A(6,5,18)$ & 119.1260 & 121.1591 & 118.9868 & 121.1591 \\
\hline$A(1,6,5)$ & 119.1271 & 124.0525 & 118.9952 & 124.0525 \\
\hline$A(1,6,26)$ & 118.6462 & 103.5659 & 118.7091 & 103.5659 \\
\hline$A(5,6,26)$ & 122.2267 & 126.7466 & 122.2956 & 126.7466 \\
\hline$A(3,7,10)$ & 121.8140 & 120.9763 & 121.9843 & 120.9763 \\
\hline$A(3,7,16)$ & 118.5427 & 119.4985 & 118.6825 & 119.4985 \\
\hline$A(10,7,16)$ & 119.6433 & 119.5252 & 119.3331 & 119.5252 \\
\hline$A(4,8,9)$ & 121.8140 & 120.9759 & 121.9845 & 120.9759 \\
\hline$A(4,8,19)$ & 118.5427 & 119.4990 & 118.6819 & 119.4990 \\
\hline$A(9,8,19)$ & 119.6433 & 119.5251 & 119.3336 & 119.5251 \\
\hline$A(8,9,10)$ & 119.5415 & 119.5096 & 119.3130 & 119.5096 \\
\hline$A(8,9,15)$ & 122.2483 & 121.6954 & 122.2921 & 121.6954 \\
\hline$A(10,9,15)$ & 118.2103 & 118.7949 & 118.3949 & 118.7949 \\
\hline$A(7,10,9)$ & 119.5415 & 119.5142 & 119.3128 & 119.5142 \\
\hline $\mathrm{A}(7,10,11)$ & 122.2483 & 121.7035 & 122.2921 & 121.7035 \\
\hline$A(9,10,11)$ & 118.2103 & 118.7822 & 118.3951 & 118.7822 \\
\hline$A(10,11,12)$ & 118.0966 & 118.0746 & 118.2627 & 118.0746 \\
\hline$A(10,11,13)$ & 121.1503 & 120.7675 & 121.0774 & 120.7675 \\
\hline $\mathrm{A}(12,11,13)$ & 120.7531 & 121.1578 & 120.6599 & 121.1578 \\
\hline$A(11,13,14)$ & 120.6395 & 120.4489 & 120.5275 & 120.4489 \\
\hline$A(11,13,20)$ & 120.4923 & 120.9732 & 120.2867 & 120.9732 \\
\hline$A(14,13,20)$ & 118.8683 & 118.5779 & 119.1858 & 118.5779 \\
\hline$A(13,14,15)$ & 120.6395 & 120.4423 & 120.5275 & 120.4423 \\
\hline$A(13,14,21)$ & 118.8683 & 118.5802 & 119.1858 & 118.5802 \\
\hline$A(15,14,21)$ & 120.4923 & 120.9774 & 120.2867 & 120.9774 \\
\hline$A(9,15,14)$ & 121.1503 & 120.7641 & 121.0775 & 120.7641 \\
\hline$A(9,15,22)$ & 118.0966 & 118.0731 & 118.2627 & 118.0731 \\
\hline$A(14,15,22)$ & 120.7531 & 121.1628 & 120.6598 & 121.1628 \\
\hline$A(1,23,24)$ & 121.8132 & 115.3929 & 121.9791 & 115.3929 \\
\hline$A(1,23,25)$ & 118.5459 & 126.8252 & 118.6997 & 126.8252 \\
\hline$A(24,23,25)$ & 119.6409 & 114.2224 & 119.3212 & 114.2224 \\
\hline$A(23,24,27)$ & 122.2478 & 126.0912 & 122.2879 & 126.0912 \\
\hline$A(23,24,28)$ & 119.5406 & 114.7844 & 119.3122 & 114.7844 \\
\hline$A(27,24,28)$ & 118.2116 & 119.0959 & 118.3999 & 119.0959 \\
\hline$A(6,26,28)$ & 121.8132 & 128.0747 & 121.9807 & 128.0747 \\
\hline$A(6,26,29)$ & 118.5459 & 107.8530 & 118.6991 & 107.8530 \\
\hline$A(28,26,29)$ & 119.6409 & 118.5913 & 119.3203 & 118.5913 \\
\hline$A(24,27,30)$ & 121.1495 & 120.5099 & 121.0732 & 120.5099 \\
\hline$A(24,27,31)$ & 118.0983 & 118.6069 & 118.2727 & 118.6069 \\
\hline$A(30,27,31)$ & 120.7522 & 120.8832 & 120.6542 & 120.8832 \\
\hline$A(24,28,26)$ & 119.5407 & 119.0596 & 119.3100 & 119.0596 \\
\hline$A(24,28,32)$ & 118.2115 & 119.2103 & 118.4002 & 119.2103 \\
\hline$A(26,28,32)$ & 122.2478 & 121.7300 & 122.2898 & 121.7300 \\
\hline$A(27,30,33)$ & 120.6389 & 120.3763 & 120.5268 & 120.3763 \\
\hline$A(27,30,34)$ & 120.4915 & 120.6477 & 120.2782 & 120.6477 \\
\hline$A(33,30,34)$ & 118.8696 & 118.9760 & 119.1950 & 118.9760 \\
\hline$A(28,32,33)$ & 121.1495 & 120.4559 & 121.0736 & 120.4559 \\
\hline$A(28,32,35)$ & 118.0983 & 118.6441 & 118.2735 & 118.6441 \\
\hline$A(33,32,35)$ & 120.7522 & 120.8999 & 120.6529 & 120.8999 \\
\hline$A(30,33,32)$ & 120.6389 & 120.3516 & 120.5263 & 120.3516 \\
\hline$A(30,33,36)$ & 118.8696 & 119.0027 & 119.1956 & 119.0027 \\
\hline$A(32,33,36)$ & 120.4915 & 120.6457 & 120.2781 & 120.6457 \\
\hline
\end{tabular}


The bond angles with the highest value at both levels of theory using the two basis sets are all in the vicinity of $120^{\circ}$ as shown in Table 2 . These include $A(32,33,36)$, $A(30,33,32), A(33,30,34), A(7,10,11), A(26,28.32)$, $A(23,24,27), \quad A(4,8,9), \quad A(4,5,6), \quad A(2,1,23)$ etc. Interestingly, all these angles are $\mathrm{C}-\mathrm{C}-\mathrm{C}$ angles within the benzene rings; thus accounting for the more symmetric shape observed in pentacene. All angles in the molecule are predicted to be more than $90^{\circ}$ and hence there is an overall spread in the molecular structure as the atoms are not close to each other.

\section{Optimized Dihedral Angles}

The structure of a molecule can be defined with high precision by the dihedral angles between three successive chemical bond vectors. The dihedral angle varies only the distance between the first and the fourth atoms; the other interatomic distances are constrained by the chemical bond lengths bond angles. The optimized dihedral angles are shown in Table 3.

\section{Table 3: Optimized Dihedral angles $\left(^{\circ}\right)$ of pentacene molecule}

\begin{tabular}{|c|c|c|c|c|}
\hline $\begin{array}{l}\text { Geometrical } \\
\text { parameter }\end{array}$ & $\begin{array}{l}\text { RHF/6-31G } \\
\text { Gas }\end{array}$ & $\begin{array}{l}\text { RHF/6-31G* } \\
\text { Gas }\end{array}$ & $\begin{array}{l}\text { B3LYP/6-31G } \\
\text { Gas }\end{array}$ & $\begin{array}{l}\text { B3LYP/6-31G } \\
\text { Gas }\end{array}$ \\
\hline
\end{tabular}

\begin{tabular}{|c|c|c|c|c|}
\hline $\mathrm{D}(6,1,2,3)$ & 0.0002 & -3.5256 & 0.0064 & -3.5256 \\
\hline$D(6,1,2,17)$ & -179.9989 & 178.3750 & -180.0176 & 178.3750 \\
\hline$D(23,1,2,3)$ & 179.9962 & 158.4370 & 179.9924 & 158.4370 \\
\hline$D(23,1,2,17)$ & -0.0028 & -19.6624 & 0.0036 & -19.6624 \\
\hline$D(2,1,6,5)$ & -0.0006 & 3.6366 & -0.0038 & 3.6366 \\
\hline$D(2,1,6,26)$ & 179.9981 & 158.5815 & 179.9914 & 158.5815 \\
\hline$D(23,1,6,5)$ & -179.9968 & -154.7266 & -179.9903 & -154.7266 \\
\hline$D(23,1,6,26)$ & 0.0019 & 0.2183 & 0.0049 & 0.2183 \\
\hline$D(2,1,23,24)$ & 179.9990 & 179.5457 & -180.0017 & 179.5457 \\
\hline$D(2,1,23,25)$ & 0.0046 & 22.2073 & 0.0159 & 22.2073 \\
\hline $\mathrm{D}(6,1,23,24)$ & -0.0049 & -22.5040 & -0.0157 & -22.5040 \\
\hline $\mathrm{D}(6,1,23,25)$ & -179.9993 & -179.8423 & 180.0019 & -179.8423 \\
\hline$D(1,2,3,4)$ & 0.0006 & 1.8585 & -0.0028 & 1.8585 \\
\hline$D(1,2,3,7)$ & 180.0001 & -178.1247 & -180.0064 & -178.1247 \\
\hline$D(17,2,3,4)$ & 179.9996 & 179.9308 & -180.0141 & 179.9308 \\
\hline$D(17,2,3,7)$ & -0.0009 & -0.0524 & -0.0176 & -0.0524 \\
\hline$D(2,3,4,5)$ & -0.0009 & 0.1839 & -0.0033 & 0.1839 \\
\hline$D(2,3,4,8)$ & 179.9995 & -179.9227 & -180.0048 & -179.9227 \\
\hline$D(7,3,4,5)$ & 179.9996 & -179.8326 & -179.9999 & -179.8326 \\
\hline$D(7,3,4,8)$ & 0.0000 & 0.0609 & -0.0014 & 0.0609 \\
\hline$D(2,3,7,10)$ & -179.9994 & -179.9531 & 180.0064 & -179.9531 \\
\hline $\mathrm{D}(2,3,7,16)$ & 0.0001 & -0.0210 & 0.0037 & -0.0210 \\
\hline$D(4,3,7,10)$ & 0.0001 & 0.0639 & 0.0028 & 0.0639 \\
\hline $\mathrm{D}(4,3,7,16)$ & 179.9996 & 179.9960 & 180.0002 & 179.9960 \\
\hline$D(3,4,5,6)$ & 0.0004 & -0.0283 & 0.0059 & -0.0283 \\
\hline$D(3,4,5,18)$ & -179.9996 & -179.9800 & 180.0141 & -179.9800 \\
\hline $\mathrm{D}(8,4,5,6)$ & -180.0000 & -179.9193 & 180.0074 & -179.9193 \\
\hline$D(8,4,5,18)$ & 0.0000 & 0.1290 & 0.0156 & 0.1290 \\
\hline $\mathrm{D}(3,4,8,9)$ & -0.0001 & -0.0966 & -0.0013 & -0.0966 \\
\hline$D(3,4,8,19)$ & -180.0001 & 180.0000 & -180.0000 & 180.0000 \\
\hline$D(5,4,8,9)$ & 180.0003 & 179.7937 & -180.0029 & 179.7937 \\
\hline $\mathrm{D}(5,4,8,19)$ & 0.0003 & -0.1098 & -0.0015 & -0.1098 \\
\hline$D(4,5,6,1)$ & 0.0003 & -2.0317 & -0.0024 & -2.0317 \\
\hline$D(4,5,6,26)$ & -179.9984 & -151.1167 & -179.9974 & -151.1167 \\
\hline$D(18,5,6,1)$ & 180.0003 & 177.9185 & -180.0105 & 177.9185 \\
\hline$D(18,5,6,26)$ & 0.0016 & 28.8335 & -0.0055 & 28.8335 \\
\hline$D(1,6,26,28)$ & 0.0027 & 26.6944 & 0.0095 & 26.6944 \\
\hline$D(1,6,26,29)$ & 179.9984 & 179.6927 & -180.0040 & 179.6927 \\
\hline$D(5,6,26,28)$ & -179.9986 & -179.2746 & 180.0045 & -179.2746 \\
\hline $\mathrm{D}(5,6,26,29)$ & -0.0029 & -26.2762 & -0.0090 & -26.2762 \\
\hline $\mathrm{D}(3,7,10,9)$ & 0.0000 & -0.1515 & -0.0016 & -0.1515 \\
\hline $\mathrm{D}(3,7,10,11)$ & 179.9999 & 179.9003 & -180.0029 & 179.9003 \\
\hline$D(16,7,10,9)$ & -179.9995 & 179.9164 & -179.9989 & 179.9164 \\
\hline $\mathrm{D}(16,7,10,11)$ & 0.0004 & -0.0317 & -0.0002 & -0.0317 \\
\hline $\mathrm{D}(4,8,9,10)$ & 0.0002 & 0.0089 & 0.0026 & 0.0089 \\
\hline$D(4,8,9,15)$ & -180.0003 & -179.9202 & 180.0018 & -179.9202 \\
\hline $\mathrm{D}(19,8,9,10)$ & -179.9998 & 179.9123 & 180.0012 & 179.9123 \\
\hline $\mathrm{D}(19,8,9,15)$ & -0.0003 & -0.0168 & 0.0005 & -0.0168 \\
\hline $\mathrm{D}(8,9,10,7)$ & -0.0002 & 0.1150 & -0.0011 & 0.1150 \\
\hline $\mathrm{D}(8,9,10,11)$ & 179.9999 & -179.9353 & -179.9999 & -179.9353 \\
\hline
\end{tabular}


Bajopas Volume 8 Number 2 December, 2015

\begin{tabular}{|c|c|c|c|c|}
\hline $\mathrm{D}(15,9,10,7)$ & 180.0003 & -179.9538 & 179.9996 & -179.9538 \\
\hline $\mathrm{D}(15,9,10,11)$ & 0.0004 & -0.0041 & 0.0008 & -0.0041 \\
\hline $\mathrm{D}(8,9,15,14)$ & -179.9999 & 72179.9107 & 180.0002 & 179.9107 \\
\hline $\mathrm{D}(8,9,15,22)$ & 0.0003 & -0.1009 & 0.0016 & -0.1009 \\
\hline $\mathrm{D}(10,9,15,14)$ & -0.0004 & -0.0189 & -0.0005 & -0.0189 \\
\hline $\mathrm{D}(10,9,15,22)$ & -180.0002 & 179.9695 & -179.9991 & 179.9695 \\
\hline $\mathrm{D}(7,10,11,12)$ & -0.0001 & -0.1060 & 0.0003 & -0.1060 \\
\hline $\mathrm{D}(7,10,11,13)$ & -180.0002 & -179.9865 & -179.9999 & -179.9865 \\
\hline $\mathrm{D}(9,10,11,12)$ & 179.9998 & 179.9455 & 179.9991 & 179.9455 \\
\hline $\mathrm{D}(9,10,11,13)$ & -0.0003 & 0.0650 & -0.0012 & 0.0650 \\
\hline $\mathrm{D}(10,11,13,14)$ & 0.0001 & -0.1018 & 0.0012 & -0.1018 \\
\hline $\mathrm{D}(10,11,13,20)$ & -180.0000 & 179.9569 & -179.9995 & 179.9569 \\
\hline $\mathrm{D}(12,11,13,14)$ & -180.0000 & -179.9786 & -179.9991 & -179.9786 \\
\hline $\mathrm{D}(12,11,13,20)$ & -0.0001 & 0.0801 & 0.0003 & 0.0801 \\
\hline $\mathrm{D}(11,13,14,15)$ & 0.0000 & 0.0780 & -0.0008 & 0.0780 \\
\hline $\mathrm{D}(11,13,14,21)$ & 180.0000 & 180.0000 & 179.9987 & 180.0000 \\
\hline $\mathrm{D}(20,13,14,15)$ & -179.9999 & -179.9793 & 179.9998 & -179.9793 \\
\hline $\mathrm{D}(20,13,14,21)$ & 0.0001 & -0.0573 & -0.0006 & -0.0573 \\
\hline$D(13,14,15,9)$ & 0.0002 & -0.0166 & 0.0005 & -0.0166 \\
\hline $\mathrm{D}(13,14,15,22)$ & 180.0000 & 179.9954 & 179.9991 & 179.9954 \\
\hline $\mathrm{D}(21,14,15,9)$ & -179.9998 & -179.9366 & -179.9991 & -179.9366 \\
\hline $\mathrm{D}(21,14,15,22)$ & 0.0000 & 0.0753 & -0.0005 & 0.0753 \\
\hline$D(1,23,24,27)$ & -179.9934 & -158.2993 & 180.0235 & -158.2993 \\
\hline $\mathrm{D}(1,23,24,28)$ & 0.0033 & 19.7293 & 0.0121 & 19.7293 \\
\hline$D(25,23,24,27)$ & 0.0010 & 1.9344 & 0.0058 & 1.9344 \\
\hline $\mathrm{D}(25,23,24,28)$ & 179.9976 & 179.9629 & -180.0057 & 179.9629 \\
\hline $\mathrm{D}(23,24,27,30)$ & -179.9981 & 177.9688 & 180.0083 & 177.9688 \\
\hline $\mathrm{D}(23,24,27,31)$ & -0.0039 & -2.0448 & -0.0098 & -2.0448 \\
\hline $\mathrm{D}(28,24,27,30)$ & 0.0052 & 0.0171 & 0.0197 & 0.0171 \\
\hline $\mathrm{D}(28,24,27,31)$ & -180.0006 & -179.9964 & 180.0016 & -179.9964 \\
\hline $\mathrm{D}(23,24,28,26)$ & 0.0014 & 1.8178 & 0.0025 & 1.8178 \\
\hline $\mathrm{D}(23,24,28,32)$ & -179.9988 & -178.1865 & -179.9971 & -178.1865 \\
\hline $\mathrm{D}(27,24,28,26)$ & 179.9982 & 179.9947 & -180.0085 & 179.9947 \\
\hline $\mathrm{D}(27,24,28,32)$ & -0.0020 & -0.0096 & -0.0082 & -0.0096 \\
\hline $\mathrm{D}(6,26,28,24)$ & -0.0044 & -29.4850 & -0.0133 & -29.4850 \\
\hline $\mathrm{D}(6,26,28,32)$ & 179.9958 & 150.5193 & 179.9864 & 150.5193 \\
\hline $\mathrm{D}(29,26,28,24)$ & 179.9999 & 179.9984 & 180.0003 & 179.9984 \\
\hline $\mathrm{D}(29,26,28,32)$ & 0.0002 & 0.0027 & -0.0001 & 0.0027 \\
\hline$D(24,27,30,33)$ & -0.0043 & -0.0110 & -0.0155 & -0.0110 \\
\hline $\mathrm{D}(24,27,30,34)$ & 179.9948 & 179.9952 & -180.0170 & 179.9952 \\
\hline$D(31,27,30,33)$ & -179.9985 & -179.9971 & 180.0031 & -179.9971 \\
\hline $\mathrm{D}(31,27,30,34)$ & 0.0007 & 0.0090 & 0.0016 & 0.0090 \\
\hline $\mathrm{D}(24,28,32,33)$ & -0.0019 & -0.0040 & -0.0075 & -0.0040 \\
\hline $\mathrm{D}(24,28,32,35)$ & -179.9982 & -179.9878 & 180.0052 & -179.9878 \\
\hline $\mathrm{D}(26,28,32,33)$ & 179.9978 & 179.9916 & -180.0072 & 179.9916 \\
\hline $\mathrm{D}(26,28,32,35)$ & 0.0016 & 0.0078 & 0.0056 & 0.0078 \\
\hline $\mathrm{D}(27,30,33,32)$ & 0.0002 & -0.0029 & -0.0008 & -0.0029 \\
\hline $\mathrm{D}(27,30,33,36)$ & 179.9998 & -179.9927 & -180.0023 & -179.9927 \\
\hline$D(34,30,33,32)$ & 180.0010 & 179.9910 & 180.0007 & 179.9910 \\
\hline $\mathrm{D}(34,30,33,36)$ & 0.0006 & 0.0013 & -0.0008 & 0.0013 \\
\hline $\mathrm{D}(28,32,33,30)$ & 0.0029 & 0.0104 & 0.0123 & 0.0104 \\
\hline $\mathrm{D}(28,32,33,36)$ & -179.9967 & -180.0000 & 180.0138 & -180.0000 \\
\hline $\mathrm{D}(35,32,33,30)$ & 179.9991 & 179.9938 & -180.0008 & 179.9938 \\
\hline$D(35,32,33,36)$ & -0.0005 & -0.0166 & 0.0008 & -0.0166 \\
\hline
\end{tabular}

As shown in Table 3, there is slight variation in the predicted dihedral angles for some bonds as we move from RHF to DFT level. However, the variation is very minimal except in some particular cases where the two basis sets predict different orientations to the angles. At both levels of theory, the angles are either too small

\section{Atomic Charge Distribution}

Within molecular system, atoms can be treated as a quantum mechanical system. On the basis of the topology of the electron density the atomic charges in (approaching zero) or almost $180^{\circ}$ similarly The dihedral angle is considered to be positive if a clockwise rotation is performed with the molecule and it will be negative when an anti clockwise rotation is performed with the molecule in its plane.

the molecule can be explained. The electrostatic potential derived charges using the CHelpG scheme of Breneman at different atomic positions in gas phase are computed at RHF and DFT levels of theory using 6-31G 
Bajopas Volume 8 Number 2 December, 2015

and 6-31G* basis sets. The Mulliken population analysis partitions the charges among the atoms of the molecule by dividing orbital overlap evenly between two atoms. Whereas the electrostatic potential derived charges assign point charges to fit the computed electrostatic potential at a number of points on or near the Van Der Waal surface. Hence, it is appropriate to consider the charges calculated by CHelpG scheme of Breneman instead of Mulliken population analysis.
Table 4 shows the electrostatic potential derived atomic charges of pentacene molecule. The charges are almost evenly distributed with the Carbon atoms retaining most of the bulk negative charge and the Hydrogen atoms retaining the bulk positive charge. This can be expected due to the free movement of electrons between the Carbon atoms in the five fused benzene rings.

Table 4: Electrostatic potential derived charges

\begin{tabular}{|c|c|c|c|c|c|}
\hline $\mathrm{S} / \mathrm{N}$ & Atom & $\begin{array}{l}\text { RHF/6-31G } \\
\text { Gas }\end{array}$ & $\begin{array}{l}\text { RHF/6-31G* } \\
\text { Gas }\end{array}$ & $\begin{array}{l}\text { B3LYP/6-31G } \\
\text { Gas }\end{array}$ & $\begin{array}{l}\text { B3LYP/6-31G* } \\
\text { Gas }\end{array}$ \\
\hline 1 & $\mathrm{C}$ & 0.062240 & 0.107974 & 0.091598 & 0.16648 \\
\hline 2 & C & -0.172484 & -0.278425 & -0.228535 & -0.30156 \\
\hline 3 & C & 0.062225 & 0.178206 & 0.091629 & 0.16649 \\
\hline 4 & C & 0.062225 & 0.141149 & 0.091630 & 0.16648 \\
\hline 5 & C & -0.172484 & -0.234382 & -0.228535 & -0.30157 \\
\hline 6 & C & -0.062240 & 0.117416 & 0.091624 & 0.16650 \\
\hline 7 & C & -0.172319 & -0.294388 & -0.225408 & -0.29822 \\
\hline 8 & C & -0.172320 & -0.295986 & -0.225401 & -0.29822 \\
\hline 9 & C & -0.066066 & 0.146512 & 0.075625 & 0.14771 \\
\hline 10 & C & -0.066066 & 0.144103 & 0.075625 & 0.14772 \\
\hline 11 & C & -0.135957 & -0.179701 & -0.143134 & -0.17949 \\
\hline 12 & $\mathrm{H}$ & 0.209939 & 0.131178 & 0.129479 & 0.13054 \\
\hline 13 & C & -0.216068 & -0.142957 & -0.136443 & -0.14152 \\
\hline 14 & C & -0.216068 & -0.283655 & -0.136443 & -0.14152 \\
\hline 15 & C & -0.135957 & 0.181247 & -0.143132 & -0.17949 \\
\hline 16 & $\mathrm{H}$ & 0.218690 & 0.134039 & 0.131364 & 0.12959 \\
\hline 17 & $\mathrm{H}$ & 0.219254 & 0.122794 & 0.131221 & 0.12946 \\
\hline 18 & $\mathrm{H}$ & 0.219254 & 0.132107 & 0.131221 & 0.12946 \\
\hline 19 & $\mathrm{H}$ & 0.218690 & 0.131107 & 0.131363 & 0.12959 \\
\hline 20 & $\mathrm{H}$ & 0.200651 & 0.131047 & 0.125608 & 0.13095 \\
\hline 21 & $\mathrm{H}$ & 0.200651 & 0.131635 & 0.125608 & 0.13095 \\
\hline 22 & $\mathrm{H}$ & 0.209939 & 0.131311 & 0.129479 & 0.13054 \\
\hline 23 & C & -0.172325 & -0.233291 & -0.225398 & -0.29822 \\
\hline 24 & C & -0.066087 & 0.106285 & 0.075585 & 0.14772 \\
\hline 25 & $\mathrm{H}$ & 0.218692 & 0.135856 & 0.131370 & 0.12959 \\
\hline 26 & C & -0.172325 & -0.273691 & -0.225431 & -0.29823 \\
\hline 27 & C & -0.135985 & -0.184688 & -0.143236 & -0.17950 \\
\hline 28 & C & -0.066087 & 0.134561 & 0.075613 & 0.14772 \\
\hline 29 & $\mathrm{H}$ & 0.218692 & 0.104486 & 0.131369 & 0.12959 \\
\hline 30 & C & -0.216062 & -0.133115 & -0.136421 & -0.14151 \\
\hline 31 & $\mathrm{H}$ & 0.209939 & 0.128114 & 0.129479 & 0.13054 \\
\hline 32 & C & -0.135985 & -0.176900 & -0.143243 & -0.17950 \\
\hline 33 & C & -0.216062 & -0.137127 & -0.136417 & -0.14151 \\
\hline 34 & $\mathrm{H}$ & 0.200652 & 0.132043 & 0.125604 & 0.13095 \\
\hline 35 & $\mathrm{H}$ & 0.209939 & 0.133349 & 0.129480 & 0.13054 \\
\hline 36 & $\mathrm{H}$ & 0.200652 & 0.131323 & 0.125605 & 0.13095 \\
\hline
\end{tabular}

\section{CONCLUSION}

The optimized structure and atomic charge distribution of the organic semi-conductor molecule pentacene has been studied using the Gaussian software package at the RHF and DFT levels of theory. Basically, it is predicted that the bond lengths are relatively short and strong with the $\mathrm{C}-\mathrm{H}$ bonds being the strongest and the $\mathrm{C}-\mathrm{C}$ bonds being the weakest. The charge distribution is fairly symmetric with the hydrogen atoms being slightly electropositive and the carbon atoms electronegative. 


\section{REFERENCES}

Byoungnam Park,(2008).Channel Formation in singlemonolayer pentacene thin film transistors electronic and structural properties of pentacene at organic/inorganic interfaces materials science thesis at the university of wisconsin-madison

Dan Kolb,(2005). Pentacene-based organic transistors, School of Engineering, University of Durham

Harada K., Riede M., Leo K., Hild O.R., and Elliott C.M. (2008)., Pentacenehomojunctions: Electron and hole transport properties and related photovoltaic responses Phys. Rev. B 77, 195212.

Hwan S.K., Young H. C., Hoon S.H and Jang J.pentacene (2004). Thin Film with Large Grain on Plastic Substrate by Organic Vapor Phase Deposition Journal of the Korean Physical Society, Vol. 45 2004. 760-762
Jacquemin D., Perpète E.A., Adamo C., (2008). Modelling the UV/visible spectrum of tetrakis(phenylethynyl)benzene . Journal of Molecular Structure 863 123-127

Jacquemin D., Preat J., Wathelet V., Marie Andre J., Perpete E.A. (2005). Substitution effects on the visible spectra of 1,4-diNHPh-9,10anthraquinonesChemical Physics Letters 405 429-433

Jan A. and Wimmer E. (1991); Optimization of Gaussian-type basis sets for local spin density functional calculations. Part I. Boron through neon, optimization technique and validation

Kitamura M., and Yasuhiko Arakawa (2008);Pentacenebased organic field-effect transistors J. Phys.: Condense. Matter20 184011.

M. Kurta, P. ChinnaBabub, N.Sundaraganesanb, M. Chinarc, M. Karabacak. Molecular structure, Vibrational UV and NBO analysis of 4chloro-7-nitrobenzofuran by DFT calculations. 